\title{
Spacetime Foam as a Quantum Thermal Bath
}

\author{
Luis J. Garay \\ Instituto de Matemáticas y Física Fundamental, CSIC, C/Serrano 121, 28006 Madrid, Spain
}

(Received 12 January 1998)

\begin{abstract}
An effective model for the spacetime foam is constructed in terms of nonlocal interactions in a classical background. In the weak-coupling approximation, the evolution of the low-energy density matrix is determined by a master equation that predicts loss of quantum coherence. Moreover, spacetime foam can be described by a quantum thermal field that, apart from inducing loss of coherence, gives rise to effects such as gravitational Lamb and Stark shifts as well as quantum damping in the evolution of the low-energy observables. [S0031-9007(98)05622-1]
\end{abstract}

PACS numbers: 04.60.-m, 03.65.Bz, 04.20.Gz, 04.70.Dy

The foamlike structure of spacetime was first suggested by Wheeler [1] and, since then, various components, such as wormholes [2,3] and virtual black holes [4], have been proposed. The quantum fluctuations of the geometry that constitute the spacetime foam should be of the same order as the geometry itself at the Planck scale. This would give rise to a minimum length [5] beyond which the geometrical properties of spacetime would be lost, while on larger scales it would look smooth and with a welldefined metric structure.

Planck length $l_{*}$ might play a role analogous to the speed of light in special relativity. In this theory, there is no physics beyond this speed limit and its existence may be inferred through the relativistic corrections to the Newtonian behavior. This would mean that a quantum theory of gravity could be constructed only on "this side of Planck's border" as pointed out by Markov [6]. In fact, the analogy between quantum gravity and special relativity is quite close: in the latter you can accelerate forever even though you will never reach the speed of light; in the former, given a coordinate frame, you can reduce the coordinate distance between two events as much as you want even though the proper distance between them will never decrease beyond Planck length (see Ref. [5], and references therein). This uncertainty relation $\Delta x \geq l_{*}$ also bears a close resemblance to the role of $\hbar$ in quantum mechanics: no matter which variables are used, it is not possible to have an action $I$ smaller than $\hbar$. Indeed, the uncertainty principle can adopt the form [7] $\Delta I \geq \hbar$.

Spacetime foam and the related lower bound to spacetime uncertainties would leave their imprint in low-energy physics. Indeed, low-energy experiments would effectively suffer a nonvanishing uncertainty coming from this lack of resolution in spacetime measurements. Then a loss of quantum coherence would be almost unavoidable [8]. It could also be expected that other effects such as transition-frequency shifts and quantum damping, characteristic of systems in a quantum environment [9], may be present. In this Letter, we in fact show that spacetime foam behaves as a quantum thermal bath with a nearly Planckian temperature.
In order to build an effective theory, we will substitute the spacetime foam, in which we possibly have a minimum length because the notion of distance is not valid at such scale, by a fixed background with low-energy fields living on it. We will perform a $3+1$ foliation of the effective spacetime that, for simplicity, will be regarded as flat, $t$ denoting the time parameter and $x$ the spatial coordinates. The gravitational fluctuations and the minimum length present in the original spacetime foam will be modeled by means of nonlocal interactions that relate spacetime points that are sufficiently close in the effective background, where a well-defined notion of distance exists. Furthermore, these nonlocal interactions will be described in terms of local interactions as follows. Let $\left\{h_{i}[t]\right\}$ be a basis of local gauge-invariant interactions at the spacetime point $(x, t)$ made out of factors of the form $l_{*}^{2 n(1+s)-4}[\phi(x, t)]^{2 n}, \phi$ being the low-energy field strength of spin $s$. As a notational convention, each index $i$ implies a dependence on the spatial position $x$; also any contraction of indices will entail an integral over spatial positions. Then, we can write the nonlocal effective interaction term in the Euclidean action as $I_{\text {int }}=\sum_{N} I_{N}$ with

$$
I_{N}=\frac{1}{N !} \int d t_{1} \cdots d t_{N} c^{i_{1} \cdots i_{N}}\left(t_{1} \cdots t_{N}\right) h_{i_{1}}\left[t_{1}\right] \cdots h_{i_{N}}\left[t_{N}\right]
$$

Here, $c^{i_{1} \cdots i_{N}}\left(t_{1} \cdots t_{N}\right)$ are dimensionless functions that vanish for relative spacetime distances larger than the length scale $r$ of the gravitational fluctuations. Furthermore, these coefficients can depend only on relative positions and not on the location of the gravitational fluctuation itself. The physical reason for this is conservation of energy and momentum: the fluctuations do not carry energy, momentum, or gauge charges. Thus, diffeomorphism invariance is preserved, at least at low-energy scales. One should not expect that at the Planck scale this invariance still holds. However, this violation of energy-momentum conservation is safely kept within Planck scale limits [10], where the processes will no longer be Markovian. Furthermore, the coefficients $c^{i_{1} \cdots i_{N}}\left(t_{1} \cdots t_{N}\right)$ will also contain a factor $\left[e^{-S(r) / 2}\right]^{N}, S(r)$ being the Euclidean action of the gravitational fluctuation, which is of the order $\left(r / l_{*}\right)^{2}$. 
Since higher-spin $s>0$ or higher-power $n>1$ interactions are suppressed by inverse powers of the lowenergy length scale $l$, we will concentrate on the mass term for scalar fields $h_{i}[t]=l_{*}^{-2} \phi\left(x^{i}, t\right)^{2}$, where now the index $i$ just keeps track of the dependence on the spatial position. A simple calculation shows that $I_{N} \sim$ $\epsilon^{N}(l / r)^{4}$, where $\epsilon=e^{-S(r) / 2}\left(r / l_{*}\right)^{4}\left(l / l_{*}\right)^{-2}$. The parameter $\epsilon$ has contributions from two different scales: on the one hand, it depends on the length scale of the gravitational fluctuations $r$ and, because of the exponential factor, it will be very small for fluctuations of few Planck lengths; on the other hand, it depends on the low-energy scale $l$ through its inverse squared and will therefore be very small far from Planck's regime. In the weak-coupling approximation, i.e., up to second order in the expansion parameter $\epsilon$, the trilocal and higher effective interactions do not contribute. The terms $I_{0}$ and $I_{1}$ are local and can be absorbed in the bare action (note that the coefficient $c$ appearing in $I_{0}$ is constant and that the coefficients $c^{i}(t)$ in $I_{1}$ cannot depend on spacetime positions because of diffeomorphism invariance). Consequently, we can write the interaction term as a bilocal term in the Euclidean action

$$
I_{\mathrm{int}}=\frac{1}{2} \int d t d t^{\prime} c^{i j}\left(t-t^{\prime}\right) h_{i}[t] h_{j}\left[t^{\prime}\right],
$$

where $c^{i j}\left(t-t^{\prime}\right)$ is of order $e^{-S(r)}$ and is concentrated within a spacetime region of size $r$. Then, the effective partition function has the form $Z=\int \mathcal{D} \phi e^{-I_{0}+I_{\text {int }}}, I_{0}$ being the bare low-energy action for the scalar field.

This bilocal effective action, when rotated back to Lorentzian spacetime, does not lead to a unitary evolution. The reason for this is that it is not sufficient to know the fields and their time derivatives at an instant of time in order to know their values at a later time: we need to know the history of the system. There exist different trajectories that arrive at a given configuration $(\phi, \dot{\phi})$. The future evolution depends on these past trajectories and not only on the values of $\phi$ and $\dot{\phi}$ at that instant of time. Therefore, the system cannot possess a well-defined Hamiltonian vector field and suffers from an intrinsic loss of predictability [11]. This can be best dealt with by writing, up to a determinant, the exponential of the interaction term as [12]

$$
e^{I_{\text {int }}} \sim \int \mathcal{D} \alpha e^{-\frac{1}{2} \int d t d t^{\prime} \gamma_{i j}\left(t-t^{\prime}\right) \alpha^{i}(t) \alpha^{j}\left(t^{\prime}\right)} e^{-\int d t \alpha^{i}(t) h_{i}[t]}
$$

Here, the continuous matrix $\gamma_{i j}\left(t-t^{\prime}\right)$ is the inverse of $c^{i j}\left(t-t^{\prime}\right)$, i.e., $\int d t^{\prime \prime} \gamma_{i k}\left(t-t^{\prime \prime}\right) c^{k j}\left(t^{\prime \prime}-t^{\prime}\right)=$ $\delta_{i}^{j} \delta\left(t-t^{\prime}\right)$. We see that $\alpha$ is a random spacetime function subject to a Gaussian distribution. At second order in $\epsilon$ and lowest order in $r / l$, the two-point correlation function is equal to $\left\langle\alpha^{i}(t) \alpha^{j}\left(t^{\prime}\right)\right\rangle=c^{i j}\left(t-t^{\prime}\right)$ and $\left\langle\alpha^{i}(t)\right\rangle=0$. Note that the Gaussian character of the distribution for the noise $\alpha$ is a consequence of the weak-coupling approximation (second order in $\epsilon$ ), which keeps only the bilocal term in the action. Higher-order terms would introduce deviations from this noise distribution. The nonunitary nature of the bilocal interaction has been encoded inside the function $\alpha$, so that, when insisting on writing the system in terms of a Hamiltonian, an additional sum over the part of the system that is unknown naturally appears. Note also that we have a single function $\alpha^{i}(t)$ because we are considering only one local interaction; we will have a different function $\alpha$ for each kind of interaction.

The Lorentzian dynamics of the low-energy field will be governed by a master equation which can be derived after a number of steps and approximations that are briefly outlined in what follows. For each fixed function $\alpha$, we first calculate the evolution equation for the density matrix $\rho_{\alpha}(t)$ obtained with the Hamiltonian

$$
H_{\alpha}(t)=H_{0}[t]+\alpha^{i}(t) h_{i}[t],
$$

$H_{0}[t]$ being the bare Hamiltonian of the low-energy field, and transform this equation into the interaction picture. We then integrate this equation between 0 and $t$ with two iterations and differentiate the result, so that the evolution equation becomes an integro-differential equation for the density matrix. Next, we perform the Gaussian average over $\alpha$ and expand the result up to second order in the parameter $\epsilon$, taking into account that $\rho_{\alpha}$ does not depend on $\alpha$ at zeroth order but only at first order in $\epsilon$, i.e., $\rho_{\alpha}=\rho+O(\epsilon)$ with $\rho=\left\langle\rho_{\alpha}\right\rangle$ (weak-coupling approximation). We also assume that $\rho(t)$ hardly changes within a correlation time $r$ (Markov approximation), so that $\rho(t+r) \sim \rho(t)$. Finally, we transform the resulting equation back to the Schrödinger picture. At the lowest order in $r / l$, the result is a master equation for the lowenergy density matrix which has the form [13]

$$
\dot{\rho}=-i\left[H_{0}, \rho\right]-\int_{0}^{\infty} d \tau c^{i j}(\tau)\left[h_{i},\left[h_{j}, \rho\right]\right] .
$$

The first term gives the Hamiltonian evolution that would also be present in the absence of fluctuations. The second term is a diffusion term which will be responsible for the loss of coherence (and the subsequent increase of entropy). It is a direct consequence of the foamlike structure of spacetime and the related existence of a minimum length.

The characteristic decoherence time $\tau_{d}$ induced by the diffusion term can be easily calculated and yields the following ratio between the decoherence time and the lowenergy length scale: $\tau_{d} / l \sim e^{S(r)}\left(r / l_{*}\right)^{-4}$. Because of the exponential factor, only the gravitational fluctuations whose size is very close to Planck length will give a sufficiently small coherence time. Slightly larger fluctuations will have a very small effect on the unitarity of the effective theory. For higher spins and/or powers of the field strength, the decoherence time increases by powers of $l / l_{*}$. For instance, if we consider interactions that mix fields with different spin, then the next relevant decoherence time corresponds to the scalar-fermion interaction 
term $\phi^{2} \bar{\psi} \psi$, which has an associated decoherence ratio $\tau_{d} / l$ proportional to $l / l_{*}$. Note that this decoherence time may be small enough for sufficiently high energies.

Let us now go a bit further and describe spacetime foam in terms of a quantum thermal bath. With this aim, we will consider a system consisting of the low-energy fields coupled to a quantum bath [9]. By comparing this system with the results obtained above for gravitational fluctuations, we will see that the latter can be substituted by a thermal bath. So, let us start with a Hamiltonian of the form

$$
H=H_{0}+H_{\text {int }}+H_{b} .
$$

$H_{0}$ is the bare Hamiltonian that represents the low-energy fields and $H_{b}$ is the Hamiltonian of a bath that, for simplicity, will be represented by a massless scalar field. The interaction Hamiltonian will be of the form $H_{\text {int }}=$ $\xi^{i} h_{i}$, where the noise operator $\xi$ is of the form $\xi^{i}(t)=$ $i \int d k \sqrt{\omega} \chi(\omega)\left[a^{+}(k) e^{i\left(\omega t-k x^{i}\right)}-\right.$ H.c. $]$. In this expression, $a$ and $a^{+}$are, respectively, the annihilation and creation operators associated with the bath, $\omega=\sqrt{k^{2}}$, and $\chi(\omega)$ represents the coupling between the system and the bath. This implies that $\xi^{i}(t)=\chi^{i j} p_{j}(t)$, with $p_{j}(t) \equiv$ $p\left(x^{j}, t\right)$ being the momentum of the bath scalar field and $\chi^{i j}=\int d k \chi(\omega) \cos \left[k\left(x^{i}-x^{j}\right)\right]$ being the coupling between the low-energy field and the bath in the position representation. The coupling $\chi(\omega)$ must be such that there exists a significant interaction with all the bath frequencies $\omega$ up to the natural cutoff $r^{-1}$. All the relevant information about the coupling is encoded in the commutation relations and the correlation function of the noise operator $\xi$.

Since the commutator of the noise operator $\xi$ at different times is a $c$ number, we can introduce the so-called commutative noise representation [9], which will allow us to compare this model with that of topological fluctuations previously described. This can be done by defining a new noise operator $\bar{\alpha}$ in the following form: $\bar{\alpha}^{i}(t) \rho\left(t^{\prime}\right) \equiv$ $\frac{1}{2}\left[\xi^{i}(t), \rho\left(t^{\prime}\right)\right]_{+}$. It is straightforward to check that the operator $\bar{\alpha}$ commutes at any time, i.e., $\left[\bar{\alpha}^{i}(t), \bar{\alpha}^{j}\left(t^{\prime}\right)\right]=$ 0 . However, this does not mean that it commutes with everything. Indeed, the commutator of $\bar{\alpha}$ with any lowenergy operator $A$ is nonvanishing and has the form

$$
\left[A^{i}(t), \bar{\alpha}^{j}\left(t^{\prime}\right)\right]=\int_{0}^{t} d \tau\left[A^{i}(t), h_{k}(\tau)\right] \dot{f}^{j k}\left(t^{\prime}-\tau\right),
$$

where

$$
\begin{gathered}
f^{i j}(\tau)=\int_{0}^{\infty} d \omega \omega^{2} G^{i j}(\omega) \cos \omega \tau, \\
G^{i j}(\omega)=\frac{\sin \omega\left|x^{i}-x^{j}\right|}{\omega\left|x^{i}-x^{j}\right|} \chi(\omega)^{2} .
\end{gathered}
$$

The commutator above vanishes for low-energy operators that are in the far past of the noise and is nonzero when they are in the near past or the future. Only in the so-called first Markov approximation the frontier among both regimes is sharply located where both noise and low-energy fields are at the same instant of time. Therefore, the function $f^{i j}(\tau)$ can be interpreted as a kind of memory function.

If we assume that the bath is in a thermal state $\rho_{b}=$ $Z^{-1} e^{-H_{b} / T}$ with a temperature $T$ and define the average of any operator $Q$ as $\langle Q\rangle \equiv \operatorname{Tr}_{b}\left(Q \rho_{b}\right)$, we can compute the correlation function $\bar{c}^{i j}\left(t-t^{\prime}\right) \equiv\left\langle\bar{\alpha}^{i}(t) \bar{\alpha}^{j}\left(t^{\prime}\right)\right\rangle$ :

$$
\bar{c}^{i j}(\tau)=\int_{0}^{\infty} d \omega \omega^{3} G^{i j}(\omega)[N(\omega)+1 / 2] \cos \omega \tau,
$$

where $N(\omega)=[\exp (\omega / T)-1]^{-1}$ is the mean occupation number of the bath corresponding to the frequency $\omega$. Also, it can be shown that the trace $\langle Q\rangle$ corresponds to a Gaussian average over $\bar{\alpha}$ only in the case that the bath is in a thermal state [9], as we are considering.

We are now ready, following similar steps to those outlined before, to write down the master equation for the low-energy density matrix. If we keep terms only up to second order in the expansion parameter $\bar{\epsilon}$ given by the product of the thermal correlation time $\tau_{\bar{\alpha}} \sim 1 / T$ of $\bar{\alpha}$, the size of the operator $h$ and the root mean square of $\bar{\alpha}$, which is of the order of $\sqrt{\bar{c}}$, and we also assume that $\tau_{\bar{\alpha}} / l \ll 1$, then the resulting equation has the same form as the classical master equation obtained above with the correlation function $c^{i j}(\tau)$ substituted for $\bar{c}^{i j}(\tau)$. From the identification of both models $(\bar{\alpha} \equiv \alpha)$, we conclude that the temperature of the heat bath is determined by the size of the gravitational fluctuations, i.e., $T \sim 1 / r$ and that $\bar{\epsilon}=\epsilon \ll 1$ (weak coupling approximation). Note also that the coupling $\chi(\omega)$ is uniquely determined by the correlation function $c^{i j}(\tau)$ by means of a suitable mode expansion. The zeroth order approximation in $r / l$ that we have made in order to compare and identify both models can be regarded as a kind of semiclassical approximation since all the quantum features of the noise have disappeared from the master equation.

We can however obtain a more general master equation, valid up to second order in $\epsilon$ and with no restriction in $r / l$, that takes into account the quantum nature of the gravitational fluctuations. These contributions will be fairly small in the low-energy regime, but may provide interesting information about the higher-energy regimes in which $l$ may be of the order of a few Planck lengths and for which the weak coupling approximation is still valid. The quantum noise effects [9] are reflected in the master equation through a term proportional to $f^{i j}(\tau)$ and another proportional to $\bar{c}^{i j}(\tau)$, both of them integrated over $\tau \in[0, \infty]$. Because of these incomplete integrals, each term provides two different kinds of contributions whose origin can be traced back to the well-known formula $\int_{0}^{\infty} d \tau e^{i \omega \tau}=\pi \delta(\omega)+P(i / \omega)$, where $P$ is the Cauchy principal part [14]. Thus, the $f$ term contains a dissipation part, necessary for the preservation of commutators, and a contribution to what can be interpreted as a gravitational Lamb shift. On the other hand, the $\bar{c}$ term gives rise to four different contributions: The already discussed diffusion term, another diffusion term originated from 
the vacuum fluctuations of the bath and that does not vanish at zero temperature, another contribution to the gravitational Lamb shift and, finally, a shift in the scalarfield oscillation frequencies that can be interpreted as a gravitational Stark effect. The size of these effects, compared with the bare evolution, can be calculated after some work: the thermal diffusion term is of order $e^{-S(r)}\left(r / l_{*}\right)^{4}$, which is the only one that survived in the previous approximations; the diffusion created by vacuum fluctuations, the damping term, and the Stark effect are smaller by a factor $r / l$; and the Lamb shift is smaller than the diffusion term by a factor $(r / l)^{2}$.

The models described in this Letter are particularly suited to the study of low-energy effects produced by simply connected topology fluctuations such as virtual black holes [4]. Indeed, it has been shown that virtual black holes can be represented from the low-energy point of view by effective interactions $h_{i}[t]$ like the ones employed here. The master equation can then be interpreted as providing the evolution of the density matrix in the presence of a bath of ubiquituous quantum topological fluctuations of the virtual-black-hole type.

Multiply connected fluctuations (with vanishing second Betti number) such as wormholes [2] can also be described as nonlocal interactions that, in the weak-coupling approximation, become bilocal. The coefficients $c^{i j}$ of this bilocal term do not depend on spacetime positions since multiply connected topology fluctuations connect spacetime points that may be far apart from each other. Diffeomorphism invariance also requires the spacetime independence of $c^{i j}$. This can also be seen by analyzing these wormholes from the point of view of the universal covering manifold, which is by definition simply connected. Here, each wormhole is represented by two boundaries located at infinity and suitably identified. This identification is equivalent to introducing coefficients $c^{i j}$ that relate the bases of the Hilbert space of wormholes in both regions of the universal covering manifold. Since $c^{i j}$ are just the coefficients in a change of basis, they will be constant. As a direct consequence, the correlation time for the functions $\alpha^{i}$ is infinite. This means that the functions $\alpha^{i}$ cannot be interpreted as noise sources that are Gaussian distributed at each spacetime point independently. Rather, they are infinitely coherent. The Gaussian distribution to which they are subject is therefore global, spacetime independent [3]. Consequently, the master equation contains no diffusion term and, actually, it predicts a unitary evolution for the density matrix. If we still try to represent wormholes by a thermal bath as we have done with localized gravitational fluctuations, we soon realize that, in order to reproduce the infinite correlation time, the couplings $\xi^{i}$ must be constant, that they must commute with every other operator and, related to these two facts, that only the zero-frequency mode of the bath can be coupled to the low-energy fields, in agreement with the result that the Gaussian distribution is spacetime independent and that the effective theory is, in this case, unitary.

Let us conclude with a brief summary. We have described spacetime foam in terms of a quantum thermal field, which induces a loss of coherence in the low-energy dynamics as well as other effects of quantum nature such as dissipation and gravitational Lamb and Stark shifts.

I am very grateful to G. A. Mena Marugán, P.F. González-Díaz, C. Barceló, C. Cabrillo, and J. I. Cirac for helpful discussions. I was supported by funds provided by DGICYT and MEC (Spain) under Contract Adjunct to the Project No. PB94-0107.

[1] J. A. Wheeler, in Relativity, Groups and Topology, edited by B.S. DeWitt and C.M. DeWitt (Gordon and Breach, New York, 1964). For a recent approach, see S. Carlip, Phys. Rev. Lett. 79, 4071 (1997).

[2] S. W. Hawking, Phys. Rev. D 37, 904 (1988).

[3] S. Coleman, Nucl. Phys. B307, 867 (1988).

[4] S. W. Hawking, Phys. Rev. D 53, 3099 (1996).

[5] L. J. Garay, Int. J. Mod. Phys. A 10, 145 (1995).

[6] M. A. Markov, Institute for Nuclear Research Report No. P-0187, Moscow, 1980; Institute for Nuclear Research Report No. P-0208, Moscow, 1981; as quoted in H.-H. Borzeszkowski and H.-J. Treder, The Meaning of Quantum Gravity (Reidel, Dordrecht, 1988).

[7] M. B. Mensky, Phys. Lett. A 155, 229 (1991); 162, 219 (1992).

[8] S. W. Hawking, Commun. Math. Phys. 87, 395 (1982).

[9] C. W. Gardiner, Quantum Noise (Springer-Verlag, Berlin, 1991).

[10] W. G. Unruh and R. M. Wald, Phys. Rev. D 52, 2176 (1995).

[11] D. A. Eliezer and R. P. Woodard, Nucl. Phys. B325, 389 (1989).

[12] J. Zinn-Justin, Quantum Field Theory and Critical Phenomena (Oxford University Press, Oxford, 1996), 3rd. ed.

[13] T. Banks, L. Susskind, and M.E. Peskin, Nucl. Phys. B244, 125 (1984).

[14] M. Reed and B. Simon, Methods of Modern Mathematical Physics I. Functional Analysis (Academic Press, New York, 1972). 